Supplement of SOIL, 2, 163-174, 2016

http://www.soil-journal.net/2/163/2016/

doi:10.5194/soil-2-163-2016-supplement

(C) Author(s) 2016. CC Attribution 3.0 License.

(c) (i)

Supplement of

\title{
Interactions between organisms and parent materials of a constructed Technosol shape its hydrostructural properties
}

M. Deeb et al.

Correspondence to: Maha Deeb (mahadeeb.y@gmail.com)

The copyright of individual parts of the supplement might differ from the CC-BY 3.0 licence. 
Table S1 Average mass of 1 liter of mixture of green waste compost (GWC) and excavated deep horizon for the different proportion of GWC, and average water mass ( $\mathrm{g}$ ) used for moistening every mixture at $80 \%$ of the water storage capacity according to its compost content $(n=16)$.

\begin{tabular}{lcccccc} 
GWC $\%$ & $0 \%$ & $10 \%$ & $20 \%$ & $30 \%$ & $40 \%$ & $50 \%$ \\
\hline Average soil mass & 1291 & 1263 & 1178 & 1114 & 1040 & 972 \\
Average water mass & 279 & 294 & 305 & 328 & 345 & 351 \\
\hline
\end{tabular}


Table S2 mean and standard error for all the treatments (C control, E earthworm, P plant, EP earth and plant), GWC: percentage of green waste compost in the mixtures $(\mathrm{V} / \mathrm{V})$, $\mathrm{e}_{0}$ : void ratio at non-swelling micropores (oven-dry) state, $v_{\mathrm{L}}$ : moisture ratio at macropore saturation, $v_{\mathrm{M}}$ : moisture ratio at micropore saturation, $v_{\mathrm{N}}$ : the moisture ratio at the shrinkage limit, $K_{\mathrm{bs}}$ : slope of the basic phase, $K_{\mathrm{re}}$ : slope of the residual phase, $K_{\mathrm{N}}$ : parameter related to shrinkage curve shape between the residual and basic phases, $K_{\mathrm{L}}$ : parameter related to shrinkage curve shape between the structural ad interpedal phases, and the void ratio at the end of the shrinkage period $\left(\mathrm{e}_{0}\right)$. The hydro-structural parameter representing the slope of the interpedal $K_{\mathrm{ip}}$ phase, the $k_{\mathrm{M}}$ parameter related to the shape of the soil shrinkage curves and $K_{\mathrm{re}}$ the slope of the residual phase was constant for all mixtures $\left(K_{\mathrm{ip}}=1\right)$, $\left(K_{\mathrm{M}}=-53\right)$ and $\left(K_{\mathrm{re}=0}\right)$.

\begin{tabular}{|c|c|c|c|c|c|c|c|c|c|}
\hline Treatments & GWC & $\mathrm{e}_{0}$ & $v_{\mathrm{L}}$ & $v_{M}$ & $v_{N}$ & $K_{\mathrm{bs}}$ & $K_{\text {st }}$ & $K_{\mathrm{N}}$ & $K_{\mathrm{L}}$ \\
\hline $\mathrm{C}$ & $0 \%$ & $0.91 \pm 0.02$ & $0.83 \pm 0.00$ & $0.66 \pm 0.00$ & $0.48 \pm 0.00$ & $0.46 \pm 0.01$ & $0.08 \pm 0.01$ & $0.24 \pm 0.03$ & $3.57 \pm 0.17$ \\
\hline $\mathrm{C}$ & $10 \%$ & $0.91 \pm 0.05$ & $0.94 \pm 0.04$ & $0.71 \pm 0.03$ & $0.51 \pm 0.02$ & $0.46 \pm 0.01$ & $0.02 \pm 0.01$ & $0.31 \pm 0.03$ & $1.26 \pm 0.19$ \\
\hline $\mathrm{C}$ & $20 \%$ & $1.08 \pm 0.02$ & $1.01 \pm 0.01$ & $0.73 \pm 0.01$ & $0.52 \pm 0.01$ & $0.34 \pm 0.03$ & $0.06 \pm 0.02$ & $0.70 \pm 0.06$ & $2.86 \pm 0.32$ \\
\hline $\mathrm{C}$ & $30 \%$ & $1.14 \pm 0.05$ & $1.06 \pm 0.02$ & $0.77 \pm 0.02$ & $0.52 \pm 0.01$ & $0.61 \pm 0.01$ & $0.15 \pm 0.06$ & $0.21 \pm 0.01$ & $0.43 \pm 0.02$ \\
\hline $\mathrm{C}$ & $40 \%$ & $1.29 \pm 0,02$ & $1.36 \pm 0.02$ & $0.99 \pm 0.01$ & $0.34 \pm 0.02$ & $0.24 \pm 0.01$ & $0.10 \pm 0.02$ & $0.28 \pm 0.00$ & $3.25 \pm 0.13$ \\
\hline $\mathrm{C}$ & $50 \%$ & $1.29 \pm 0.05$ & $1.32 \pm 0.03$ & $1.04 \pm 0.02$ & $0.28 \pm 0.01$ & $0.25 \pm 0.02$ & $0.02 \pm 0.01$ & $0.25 \pm 0.03$ & $2.00 \pm 0.00$ \\
\hline $\mathrm{E}$ & $0 \%$ & $0.98 \pm 0.02$ & $0.95 \pm 0.02$ & $0.73 \pm 0.01$ & $0.46 \pm 0.01$ & $0.41 \pm 0.00$ & $0.02 \pm 0.00$ & $0.22 \pm 0.01$ & $1.31 \pm 0.00$ \\
\hline $\mathrm{E}$ & $10 \%$ & $1.10 \pm 0.02$ & $0.97 \pm 0.01$ & $0.74 \pm 0.01$ & $0.48 \pm 0.01$ & $0.40 \pm 0.00$ & $0.07 \pm 0.01$ & $0.24 \pm 0.01$ & $2.75 \pm 0.13$ \\
\hline $\mathrm{E}$ & $20 \%$ & $1.14 \pm 0.05$ & $1.05 \pm 0.01$ & $0.72 \pm 0.00$ & $0.47 \pm 0.01$ & $0.37 \pm 0.02$ & $0.13 \pm 0.02$ & $0.38 \pm 0.04$ & $0.88 \pm 0.36$ \\
\hline $\mathrm{E}$ & $30 \%$ & $1.29 \pm 0.02$ & $1.14 \pm 0.02$ & $0.79 \pm 0.01$ & $0.47 \pm 0.01$ & $0.35 \pm 0.00$ & $0.17 \pm 0.00$ & $0.20 \pm 0.00$ & $1.42 \pm 0.05$ \\
\hline E & $40 \%$ & $1.28 \pm 0.04$ & $1.26 \pm 0.04$ & $1.06 \pm 0.03$ & $0.42 \pm 0.02$ & $0.27 \pm 0.00$ & $0.13 \pm 0.05$ & $0.13 \pm 0.01$ & $1.25 \pm 0.13$ \\
\hline $\mathrm{E}$ & $50 \%$ & $1.30 \pm 0.03$ & $1.35 \pm 0.03$ & $1.16 \pm 0.02$ & $0.38 \pm 0.01$ & $0.26 \pm 0.01$ & $0.08 \pm 0.04$ & $0.20 \pm 0.01$ & $1.62 \pm 0.24$ \\
\hline $\mathrm{P}$ & $0 \%$ & $0.94 \pm 0.02$ & $0.93 \pm 0.01$ & $0.75 \pm 0.01$ & $0.51 \pm 0.01$ & $0.41 \pm 0.02$ & $0.05 \pm 0.02$ & $0.26 \pm 0.02$ & $1.15 \pm 0.14$ \\
\hline $\mathrm{P}$ & $10 \%$ & $1.00 \pm 0.05$ & $1.00 \pm 0.03$ & $0.76 \pm 0.01$ & $0.51 \pm 0.01$ & $0.42 \pm 0.03$ & $0.11 \pm 0.02$ & $0.22 \pm 0.00$ & $1.25 \pm 0.13$ \\
\hline $\mathrm{P}$ & $20 \%$ & $1.15 \pm 0.02$ & $1.05 \pm 0.02$ & $0.73 \pm 0.00$ & $0.45 \pm 0.01$ & $0.31 \pm 0.04$ & $0.18 \pm 0.01$ & $0.48 \pm 0.06$ & $0.55 \pm 0.14$ \\
\hline $\mathrm{P}$ & $30 \%$ & $1.24 \pm 0.05$ & $1.23 \pm 0.01$ & $0.84 \pm 0.01$ & $0.46 \pm 0.01$ & $0.32 \pm 0.01$ & $0.16 \pm 0.01$ & $0.20 \pm 0.00$ & $1.11 \pm 0.16$ \\
\hline $\mathrm{P}$ & $40 \%$ & $1.29 \pm 0.01$ & $1.38 \pm 0.01$ & $0.96 \pm 0.01$ & $0.48 \pm 0.01$ & $0.38 \pm 0.02$ & $0.13 \pm 0.00$ & $0.12 \pm 0.01$ & $1.00 \pm 0.00$ \\
\hline $\mathrm{P}$ & $50 \%$ & $1.60 \pm 0.06$ & $1.48 \pm 0.02$ & $1.02 \pm 0.01$ & $0.31 \pm 0.01$ & $0.26 \pm 0.01$ & $0.14 \pm 0.01$ & $0.20 \pm 0.00$ & $2.00 \pm 0.20$ \\
\hline $\mathrm{EP}$ & $0 \%$ & $1.16 \pm 0.03$ & $0.96 \pm 0.00$ & $0.69 \pm 0.00$ & $0.42 \pm 0.02$ & $0.41 \pm 0.02$ & $0.13 \pm 0.03$ & $0.26 \pm 0.01$ & $1.05 \pm 0.11$ \\
\hline EP & $10 \%$ & $1.41 \pm 0.11$ & $0.97 \pm 0.01$ & $0.76 \pm 0.01$ & $0.40 \pm 0.02$ & $0.39 \pm 0.04$ & $0.00 \pm 0.00$ & $0.56 \pm 0.14$ & $1.18 \pm 0.32$ \\
\hline $\mathrm{EP}$ & $20 \%$ & $1.13 \pm 0.03$ & $1.07 \pm 0.02$ & $0.81 \pm 0.00$ & $0.47 \pm 0.01$ & $0.33 \pm 0.01$ & $0.12 \pm 0.01$ & $0.71 \pm 0.06$ & $1.00 \pm 0.00$ \\
\hline $\mathrm{EP}$ & $30 \%$ & $1.14 \pm 0.04$ & $1.16 \pm 0.01$ & $0.84 \pm 0.01$ & $0.44 \pm 0.01$ & $0.25 \pm 0.01$ & $0.10 \pm 0.02$ & $0.70 \pm 0.00$ & $1.30 \pm 0.21$ \\
\hline $\mathrm{EP}$ & $40 \%$ & $1.59 \pm 0.08$ & $1.38 \pm 0.03$ & $0.93 \pm 0.01$ & $0.44 \pm 0.03$ & $0.43 \pm 0.03$ & $0.11 \pm 0.01$ & $0.24 \pm 0.03$ & $0.88 \pm 0.02$ \\
\hline EP & $50 \%$ & $1.91 \pm 0.04$ & $1.70 \pm 0.05$ & $0.98 \pm 0.03$ & $0.30 \pm 0.01$ & $0.36 \pm 0.04$ & $0.15 \pm 0.02$ & $0.31 \pm 0.05$ & $1.10 \pm 0.05$ \\
\hline
\end{tabular}


Table S3 mean and standard error (sd) for total plant biomass ( $\mathrm{g}$ ) and available volumetric water content $\left(\mathrm{cm}^{3}{ }_{\text {water }} \mathrm{cm}^{-3}\right.$ soil $)$, the results and sd of both equations summarizing the relation between total dried plant biomass $(\mathrm{X}, \mathrm{g})$ and plant available water content $\left(\theta_{\text {Total }}, \mathrm{cm}^{3}{ }_{\text {water }} \cdot \mathrm{cm}^{-3}{ }_{\text {soil }}\right)$ were: $\mathrm{X}=8.97 . \theta_{\text {Total }}+4.07$ and $\mathrm{X}=8.97 . \theta_{\text {Total }}+$ 2.69 with and without earthworms.

\begin{tabular}{|c|c|c|c|c|c|c|c|c|}
\hline GWC & $\begin{array}{c}\theta_{\text {Total without }} \\
\text { earthworm } \\
\left(\mathrm{cm}^{-3} \cdot \mathrm{cm}^{-3}\right)\end{array}$ & $\begin{array}{l}\text { Total plant } \\
\text { biomass } \\
\text { without } \\
\text { earthworm } \\
\quad(\mathrm{g})\end{array}$ & $\begin{array}{l}\text { Prediction } \\
\text { total plant } \\
\text { biomass } \\
\text { without } \\
\text { earthworm } \\
\quad(\mathrm{g}) \\
\end{array}$ & $\begin{array}{l}\text { sd } \\
\text { prediction } \\
\text { equation }\end{array}$ & $\begin{array}{l}\theta_{\text {Total }} \text { with } \\
\text { earthworm } \\
\left(\mathrm{cm}^{-3} \cdot \mathrm{cm}^{-3}\right)\end{array}$ & $\begin{array}{c}\text { Total plant } \\
\text { biomass with } \\
\text { earthworm (g) }\end{array}$ & $\begin{array}{l}\text { Prediction } \\
\text { total plant } \\
\text { biomass } \\
\text { with } \\
\text { earthworm } \\
\quad(\mathrm{g}) \\
\end{array}$ & $\begin{array}{c}\text { sd } \\
\text { prediction } \\
\text { equation }\end{array}$ \\
\hline 0 & $0.19 \pm 0.01$ & $4.62 \pm 0.19$ & 4.25 & 0.39 & $0.22 \pm 0.02$ & $6.37 \pm 0.43$ & 5.62 & 0.39 \\
\hline 10 & $0.21 \pm 0.02$ & $4.48 \pm 0.26$ & 4.63 & 0.39 & $0.23 \pm 0.01$ & $6.14 \pm 0.47$ & 6.00 & 0.39 \\
\hline 20 & $0.25 \pm 0.02$ & $4.32 \pm 0.20$ & 5.01 & 0.38 & $0.27 \pm 0.03$ & $5.86 \pm 0.19$ & 6.38 & 0.38 \\
\hline 30 & $0.29 \pm 0.01$ & $4.93 \pm 0.12$ & 5.39 & 0.38 & $0.30 \pm 0.01$ & $6.03 \pm 0.24$ & 6.76 & 0.38 \\
\hline 40 & $0.33 \pm 0.01$ & $6.35 \pm 0.19$ & 5.77 & 0.39 & $0.34 \pm 0.05$ & $6.94 \pm 0.42$ & 7.14 & 0.39 \\
\hline 50 & $0.39 \pm 0.01$ & $6.48 \pm 0.31$ & 6.15 & 0.39 & $0.41 \pm 0.01$ & $8.07 \pm 0.38$ & 7.52 & 0.39 \\
\hline
\end{tabular}

